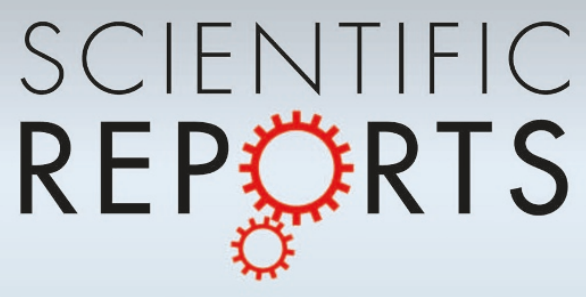

OPEN Increased cortical responses to forepaw

SUBJECT AREAS:

SENSORY PROCESSING

CORTEX stimuli immediately after peripheral deafferentation of hindpaw inputs

\author{
D. Humanes-Valera, G. Foffani \& J. Aguilar
}

Received

4 September 2014

Hospital Nacional de Parapléjicos, SESCAM, Toledo, Spain.

Accepted

12 November 2014

Published

2 December 2014

Correspondence and requests for materials should be addressed to

J.A. (jdaguilar@

sescam.jccm.es)

Both central and peripheral injuries of the nervous system induce dramatic reorganization of the primary somatosensory cortex. We recently showed that spinal cord injuries at thoracic level in anesthetized rats can immediately increase the responses evoked in the forepaw cortex by forepaw stimuli (above the level of the lesion), suggesting that the immediate cortical reorganization after deafferentation can extend across cortical representations of different paws. Here we show that a complete deafferentation of inputs from the hindpaw induced by injury or pharmacological block of the peripheral nerves in anesthetized rats also increases the responses evoked in the forepaw cortex by forepaw stimuli. This increase of cortical responses after peripheral deafferentation is not associated with gross alterations in the state of cortical spontaneous activity. The results of the present study, together with our previous works on spinal cord injury, suggest that the forepaw somatosensory cortex is critically involved in the reorganization that starts immediately after central or peripheral deafferentation of hindpaw inputs.

$\mathrm{n}$ the nervous system, both peripheral and central injuries produce cortical reorganization, which is reflected in functional and plastic changes in the long-term. Frequently, the cortical changes induced by central or peripheral deafferentation can contribute to the development of pathologies such as phantom sensations $s^{1,2}$ or neuropathic pain ${ }^{3-5}$. To fully understand the mechanisms leading to long-term cortical reorganization, it is necessary to study the changes that occur in the cortex immediately after the deafferentation.

Focusing in models of spinal cord injury, we recently characterized the cortical responses to peripheral stimulation above the level of the lesion immediately after a complete thoracic transection ${ }^{6,7}$ or thoracic hemisection $^{8}$. Our results show an increment in the amplitude of the responses evoked in the somatosensory cortex by stimuli delivered to the forepaw, above the lesion level. These changes in the responses have both a statedependent component, due to a global change in cortical state ${ }^{6}$, and a state-independent component, due to more classical unmasking mechanisms at cortico-subcortical level ${ }^{7,8}$. Interestingly, these increased responses to forepaw stimuli are not observed only in the hindpaw cortex, which is deafferented by the thoracic spinal cord injury, but also in the forepaw cortex, which would be classically considered as non-deafferented. However, in control animals the hindpaw cortex does display small responses to forepaw stimuli ${ }^{6,9,10}$, suggesting that the loss of input from the hindpaw produces a partial deafferentation also of the forepaw cortex. If this interpretation is correct, an increment in the responses evoked in the forepaw cortex by forepaw stimuli should be observed not only after central hindpaw deafferentation due to spinal cord injury, as in our previous studies, but also immediately after peripheral hindpaw deafferentation due to nerve injury. This hypothesis is supported by numerous classical studies showing immediate reorganization between the deafferented cortex and surrounding cortical areas after peripheral nerve injuries or amputations ${ }^{11-16}$. As far as we know, however, whether this immediate cortical reorganization after peripheral nerve injury can extend as far as across the cortical representations of different paws remains unknown.

To test this hypothesis we performed experiments in 29 male anesthetized rats, which were divided in three groups (Fig. 1A,B): (1) animals that received a peripheral injury to the hindpaw, consisting of a complete section of both the sciatic and saphenous nerves $(n=11) ;(2)$ animals that received a pharmacological block of the same peripheral nerves by injection of lidocaine $(n=10)$; (3) sham animals $(n=8)$. We specifically investigated the possible changes in the responses evoked in the forepaw cortex ${ }^{17}$ by electrical stimuli delivered to the forepaw, as well as possible changes in cortical spontaneous activity, within two hours after injury or pharmacological block of the peripheral nerves in the hindpaw. 
A

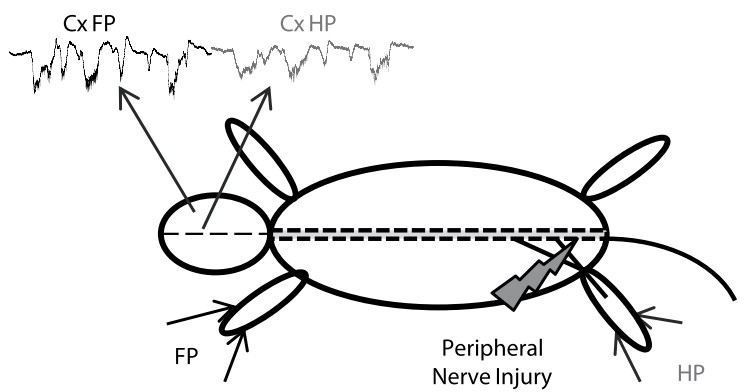

B

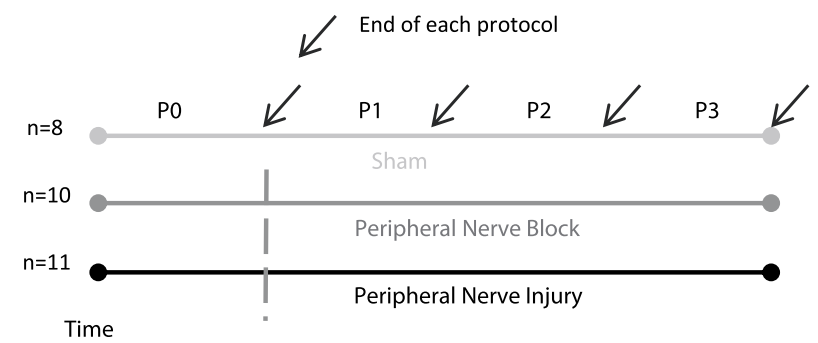

C

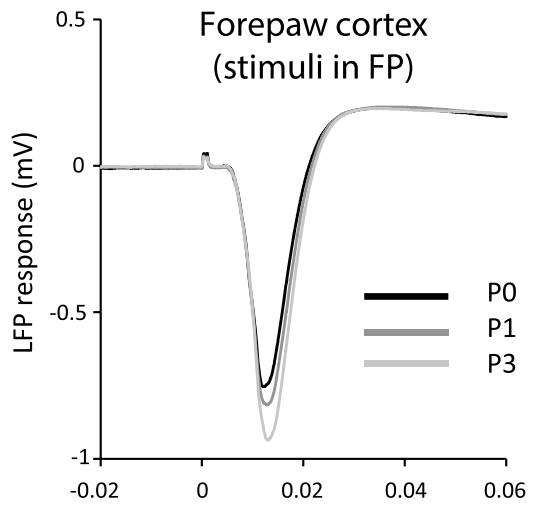

Time (s)

\section{Evoked responses}
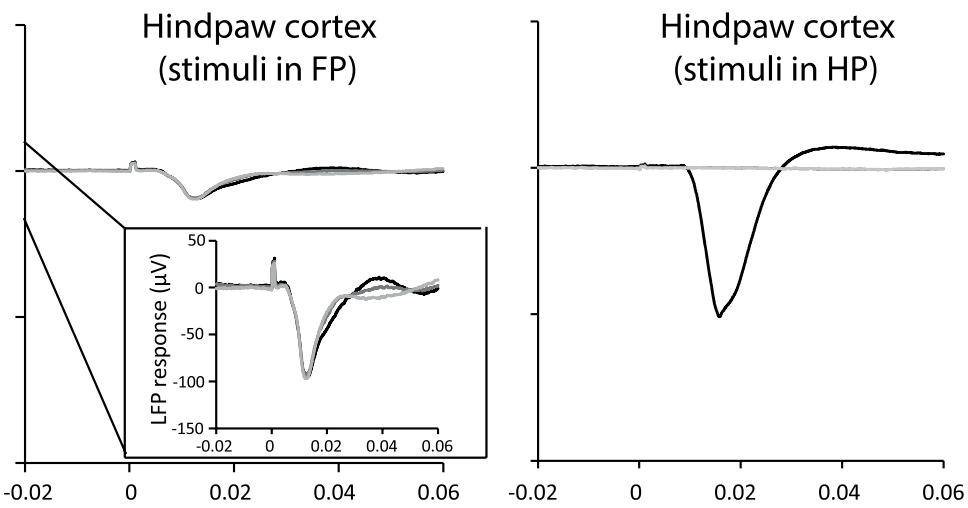

D Evolution of evoked responses in FP cortex
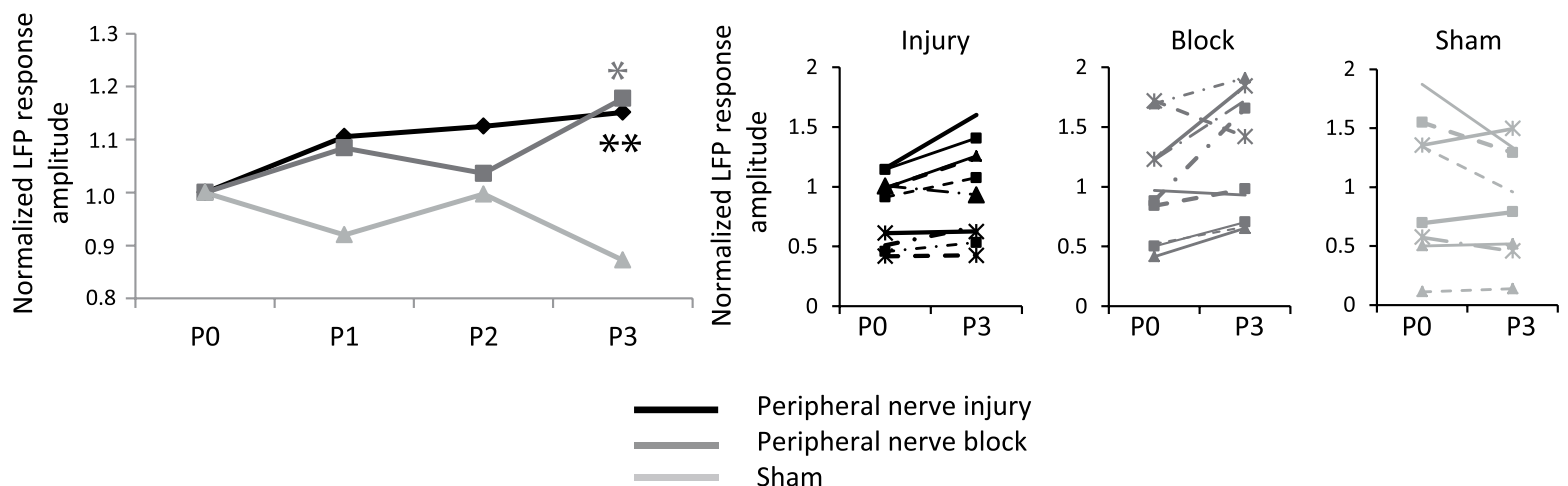

Peripheral nerve injury
Peripheral nerve block
Sham

Figure 1 Experimental protocol and evoked responses. (A) Extracellular recordings were made in the forepaw (FP) and hindpaw (HP) representations of the primary somatosensory cortex $(\mathrm{Cx})$ in urethane-anaesthetized rats. Peripheral nerve injury or pharmacological block was performed to both the saphenous and sciatic nerves. (B) We studied both the spontaneous activity and the responses evoked by electrical stimuli delivered to the hindpaw and forepaw at high intensity $(5 \mathrm{~mA})$ before (P0: control protocol with intact animal) and after deafferentation at three different time points (P1, P2 and P3: 10-20 min, 1 hour and 2 hours after deafferentation respectively). The experiment was performed in three groups of animals: animals that received injury of the peripheral nerves (black), animals that received pharmacological blockade of the peripheral nerves (dark gray), sham animals with intact nerves (light gray). (C) Grand average of local field potential (LFP) responses evoked by high intensity (5 mA) stimuli delivered either to the contralateral forepaw (left: forepaw cortex; center: hindpaw cortex, enlarged in the inset) or hindpaw (right: hindpaw cortex) before (P0: black) and after peripheral nerve injury, (P1: dark grey; P3: light grey). (D) Left: evolution of the amplitude of the responses evoked in forepaw cortex by forepaw stimuli in the different group of animals (peripheral nerve injury: black; peripheral nerve block: dark grey; sham: light grey) over time (P1, P2 and P3). *:p <0.05; ${ }^{* *}: \mathrm{p}<0.01$. Right: Corresponding variability between animals. Note: the values of the evoked responses shown in Figure $1 \mathrm{D}$ are normalized in relation to value of the response in P0 (one subject in the injury group with very high values was excluded from the variability plot but included in the average and in the analyses). The responses evoked in the forepaw cortex by forepaw stimuli significantly increased after peripheral deafferentation of hindpaw inputs. 


\section{Results}

Cortical LFP responses to forepaw and hindpaw stimuli in normal animals. We first studied the cortical evoked responses, measured by the local field potentials (LFPs) as in our previous works ${ }^{6,718,19}$. In intact animals (P0), the responses evoked in the forepaw cortex by forepaw stimuli displayed greater amplitude than the responses evoked in the hindpaw cortex by hindpaw stimuli (Fig. 1C, Table 1). Forepaw stimuli also evoked responses in the hindpaw cortex, which were almost one order of magnitude smaller than the responses evoked in the forepaw cortex (Fig. 1C, Table 1). Similarly, hindpaw stimuli also evoked small responses in the forepaw cortex (Table 1). These observations are consistent with our previous results ${ }^{6,7,10}$.

Increased responsiveness of the forepaw cortex to forepaw stimuli after peripheral deafferentaiton of hindpaw inputs. Immediately after peripheral nerve injury or pharmacological block of axonal conduction of sciatic and saphenous nerves, the responses evoked at cortical level by hindpaw stimuli were abolished, confirming that the lesion/block of saphenous and sciatic nerves was complete (Fig. 1C, Table 1). The amplitudes of LFP responses evoked in the forepaw cortex by forepaw stimuli changed in time depending on the experimental group (two-way mixed ANOVA, interaction TIME $\mathrm{x}$ GROUP: $\mathrm{F}(6,78)=3.2, \mathrm{p}=0.0073)$, significantly increasing both after nerve injury (follow-up one-way ANOVA: $\mathrm{F}(3,30)=4.59, \mathrm{p}=0.0093$ ) and after pharmacological block $(\mathrm{F}(3,27)=3.25, \mathrm{p}=0.0373)$, but not in sham animals $(\mathrm{F}(3,21)=1.64, \mathrm{p}=0.21$; Fig. 1C,D; Table 1). Specifically, the increased LFP responses reached significance in the third protocol, both after the injury (Dunnett post-hoc test: $\mathrm{p}=0.0027)$ and after the pharmacological block $(\mathrm{p}=0.0178)-$ despite partial recovery of hindpaw responses after block (Table 1). Note that the results on animals that received nerve injury remain significant after over-conservative Bonferroni correction (i.e. $\mathrm{p}<0.05 / 3=0.0166$ ). When animals that received nerve injury or pharmacological block were pooled together (one-way ANOVA; $\mathrm{F}(3,60)=7.3, \mathrm{p}=0.0003)$ the increased responsiveness reached significance already in the first protocol after deafferentation (Dunnett test; $p=0.0289$ ), transiently lost significance in the second protocol $(p=0.12)$ and was again highly significant in the third protocol $(\mathrm{p}<0.0001)$. These findings confirm our hypothesis that hindpaw peripheral deafferentation affects the responses evoked in the forepaw cortex by forepaw stimuli.

Local origin of the increased cortical responses. Because LFPs can in principle be influenced by distant sinks and sources, one might wonder whether the increased forepaw LFP responses might reflect far-field alterations that are really occurring in the deafferented hindpaw cortex. Even though the high impedance of our recording electrodes make this possibility unlikely, we explicitly ruled it out by quantifying the amplitude of LFP responses evoked in the hindpaw cortex by forepaw stimuli. In animals that received nerve injury or block $(n=21)$, the amplitude of hindpaw LFP responses to forepaw stimuli showed only a tendency to increase in the third protocol after deafferentation (paired t-test, $p=0.09$; Fig. 1C; Table 1) and the average net response increase in the hindpaw cortex $(0.02+1$ $-0.05 \mathrm{mV}$ ) was one order of magnitude smaller than the average net response increase in the forepaw cortex $(0.22+/-0.24 \mathrm{mV})$. We also confirmed the main result of increased responsiveness of the forepaw cortex to forepaw stimuli in the third protocol after nerve injury/block by quantifying the responses from the rectified multiunit activity (rMUA) of the forepaw cortex (P0: $5.6+/-3.9$ arbitrary units; P3: $6.4+/-4.2$ a.u.; paired-t test: $p=0.0255 ; n=21)$. These results support the local origin of the increased responsiveness of the forepaw cortex to forepaw stimuli after deafferentation of hindpaw inputs.

The increased cortical responsiveness is not due to gross changes in the state of cortical spontaneous activity. The observed increase in cortical responses could be due, at least in part, to a decreased cortical spontaneous activity ${ }^{6-8}$. To test if this was the case, cortical spontaneous activity was measured from the rMUA. We did not observe any changes in cortical spontaneous activity (three-way ANOVA; factor TIME and interactions with TIME: $\mathrm{F}<1.2$, $\mathrm{p}>0.33$ ). To verify that the absence of changes in cortical spontaneous activity was not due to a lack of statistical power, we repeated the analyses with progressively less conservative approaches. We did not find any statistical difference either when only "injury" and "block" experiments (i.e. without "sham" experiments) were analyzed together (three-way ANOVA: $p>0.25$ ), or separately (two-way ANOVAs: $\mathrm{p}>0.33$ ). Furthermore, when hindpaw cortex and forepaw cortex were analyzed separately in each experimental group, in "block" experiments we did not find any difference in either cortex (one-way ANOVAs; $p>0.29$ ), in "injury" experiments we did not find any difference in the hindpaw cortex $(\mathrm{p}=0.75)$, whereas the spontaneous activity tended to increase - rather than decrease - in the forepaw cortex $(\mathrm{F}(3,30)=2.9, \mathrm{p}=0.0508)$ : compared to baseline $(6.8+/-1.3 \mathrm{uV})$, spontaneous activity tended to increase in the first protocol $(7.5+/$ $-2.0 \mathrm{uV}$; Dunnett, $\mathrm{p}=0.07)$, increased in the second protocol $(7.6+/$ $-2.3 \mathrm{uV} ; \mathrm{p}=0.0278)$ but not in the third protocol $(7.3+/-1.5 \mathrm{uV}$; $\mathrm{p}=0.26)$. Finally, in 8 of 11 animals that received peripheral nerve injury, after the third protocol post nerve injury, we performed a complete transection of the spinal cord at thoracic level (T9/T10), with the same techniques as in our previous works ${ }^{6,7}$. The spinal cord injury induced the expected decrease in cortical spontaneous activity $^{6}$, as measured by a decrease mean rMUA (two-way ANOVA; PRE-POST spinal lesion: $F(1,7)=24.5, p=0.0017)$. These observations confirm that the absence of changes in cortical spontaneous activity after nerve injury or pharmacological block was not due to a lack of statistical power. Therefore, the increased responses evoked in the forepaw cortex by forepaw stimuli after peripheral deafferentation of hindpaw inputs were not due to a gross decrease of cortical spontaneous activity.

\section{Discussion}

The main result of the present work is that a complete deafferentation of hindpaw inputs induced by injury or pharmacological block of the peripheral nerves increases the responses evoked in the forepaw cortex by forepaw stimuli (above the level of the lesion) in anesthetized rats. There is a vast literature about cortical reorganization after peripheral injury (amputation or nerve injury) with altered cortical responses observed in the deafferented cortex or surrounding areas, both in the short-term ${ }^{11-16}$ and in the long-term ${ }^{20-24}$. Our results show that the immediate cortical reorganization after peripheral deafferentation can extend as far as across cortical representations of different paws in the primary somatosensory cortex.

Our finding that a peripheral loss of inputs from the hindpaw can affect cortical forepaw responses might appear surprising at first glance. However, increasing evidence shows that in normal conditions the hindpaw cortex can be activated by forepaw stimuli, as measured by both extracellular electrophysiological techniques ${ }^{9}$ and voltage-sensitive dye imaging ${ }^{10,25,26}$. Specifically, high-intensity stimulation of one extremity (forepaw or hindpaw) produces an intense response in the focus of the somatotopical corresponding place, and a gradient of weaker signal of neuronal activity that reaches the cortical representation of the other extremity ${ }^{10}$. A loss of hindpaw inputs thus represents a partial deafferentation of the forepaw cortex and can consequently alter cortical forepaw responses, as shown in our previous works with spinal cord injury ${ }^{7,8}$ and in the present work with peripheral deafferentation.

Several mechanisms could contribute to the early cortical reorganization after deafferentation. Growth of new connections due to axonal sprouting in the deafferented cortex were observed as early as few hours after retinal lesions in monkeys ${ }^{27}$ and after whisker 


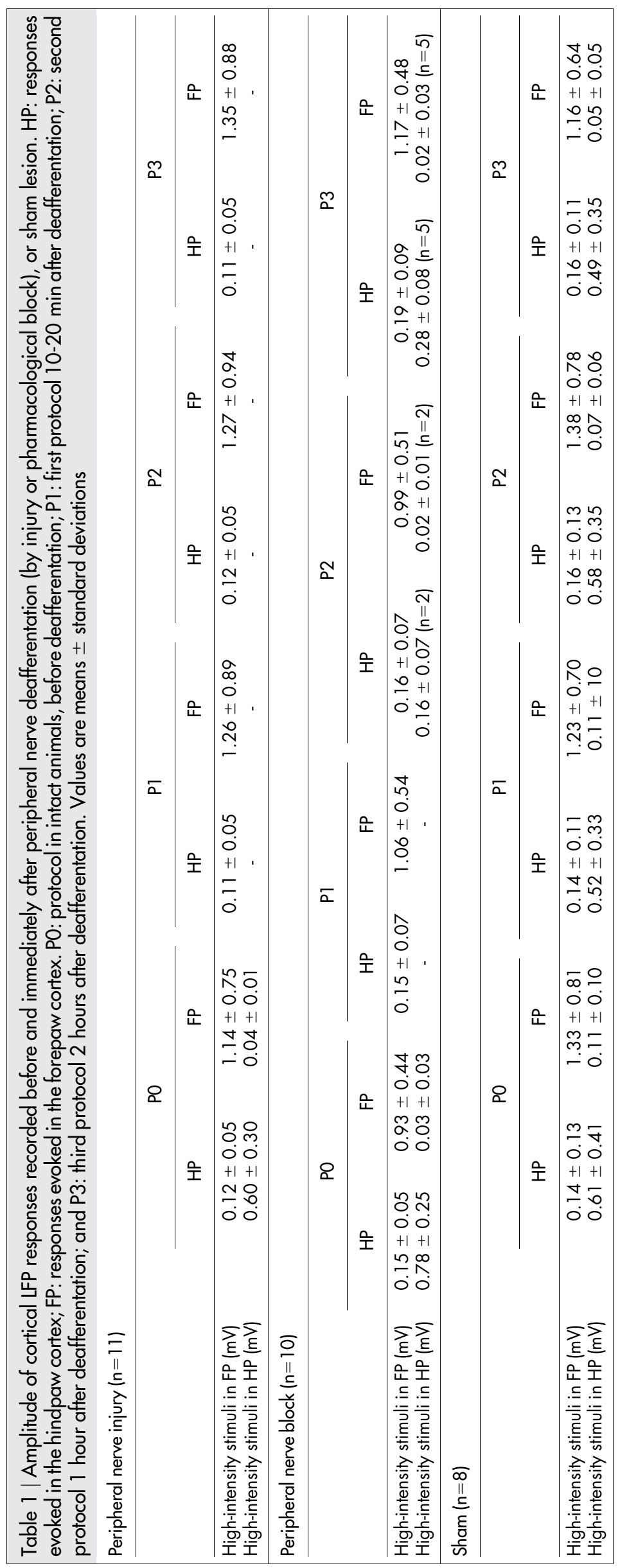


plucking in rats ${ }^{28}$. Furthermore, the loss of dendritic spines and axonal buttons of inhibitory interneurons in the deafferented cortex observed as early as $6 \mathrm{~h}$ after retinal lesion ${ }^{29}$ and the heterogeneous spine loss reported 3 days after spinal cord injury ${ }^{26}$ point toward possible changes in the excitation/inhibition ratio. Even though the time window in which our changes take place is faster, within $2 \mathrm{~h}$ after lesion, we cannot exclude that similar mechanisms could contribute to our results. Another classical possible explanation for our findings is the unmasking of latent synapses, either at cortical level ${ }^{13,30,31}$ or subcortical level ${ }^{32-35}$ possibly associated with changes in the neuromodulatory regulation at thalamo-cortical level ${ }^{36}$.

It is interesting to note that the progressive increase of forepaw responses observed here after peripheral deafferentation seems more similar to the progressive increase observed after spinal thoracic hemisection $^{8}$ than to the immediate increase observed after spinal thoracic transection ${ }^{7}$. This suggests that not only the magnitude, but also the temporal aspects of cortical reorganization might critically depend on the extent of the deafferentation. Progressive increase in cortical excitability might therefore be a common feature in partial deafferentation models. It is tempting to speculate that the tendency of increased spontaneous activity in the forepaw cortex described in our data might have contributed to the delayed increase of cortical responses after nerve injury (but note that this consideration does not apply to "block" experiments). Indeed, even though we can confidently exclude gross decreases of cortical spontaneous activity, the increased cortical responses could be mechanistically associated with subtler changes in spontaneous activity ${ }^{18}$.

Central and peripheral deafferentation of hindpaw inputs can produce similar increase in forepaw evoked responses with different effects in cortical spontaneous activity. Further investigations of similarities and differences in cortical reorganization after central or peripheral injuries might have translational value to shed light into the common (or different) processes leading to long-term development of neuropathic pain after somatosensory deafferentation. So far, the results of the present study together with our previous work suggest that the forepaw somatosensory cortex is critically involved in the reorganization that starts immediately after the central or peripheral loss of hindpaw inputs.

\section{Methods}

All experiments were performed following the rules of International Council for Laboratory Animal Science and the European Union regulation 2010/63/EU. The experimental methods were carried out in accordance with the approved guidelines of the Ethical Committee for Animal Research of the Hospital Nacional de Parapléjicos (Toledo, Spain).

Experimental procedures. The main experimental protocol was performed in the group of animals that received peripheral nerve injury $(n=11)$. Animals were anesthetized with urethane $(1.5 \mathrm{~g} / \mathrm{kg}$, i.p.) and placed in a stereotaxic frame (SR-6 Narishige Scientific Instruments, Tokyo, Japan). Body temperature was kept at $36.5^{\circ} \mathrm{C}$. Lidocaine $2 \%$ was applied over the body surface in contact with the frame and over the shaved areas for incisions. The skin of the lumbar zone (L1-S1) was softly removed and the sciatic and saphenous nerves on the left side of the body were exposed. A craniotomy was performed on the right side of the midline over the somatosensory cortex (antero-posterior: -2 to 2; medio-lateral: 1-5; ref. 17) and the cisterna magna was opened. The recording electrodes (tungsten electrodes with $4-$ $5 \mathrm{M} \Omega$ impedance at $1 \mathrm{kHz}$ ) were placed in the infragranular layers of the hindpaw and forepaw representations of the primary somatosensory cortex as described in our previous works $\mathrm{s}^{6,7,18,19}$.

We performed the control protocol (P0), which allowed us to record cortical evoked responses to stimulation of both extremities (forepaw and hindpaw) and cortical spontaneous activity with the intact animal (Fig. 1A,B). We then sectioned the sciatic and saphenous nerves with a scissor, guided by a surgical microscope to ensure a complete section of both nerves without damaging the surrounding tissue and irrigation of the area. Immediately after the injury, few electrical stimuli at very-high intensity $(10 \mathrm{~mA})$ were applied to the deafferented hindpaw to confirm that no physiological responses were evoked in the cortex. Electrophysiological recordings were continuously acquired during the injury to confirm the stability of the recordings. The stimulation protocols were applied at three different times after the injury: 10-20 min (P1), 1 hour (P2) and 2 hours (P3).

The stimulation protocol consisted of a train of electrical pulses (100 stimuli at $0.5 \mathrm{~Hz}$ ) applied subcutaneously in the wrist of the forepaw and the ankle in the hindpaw similarly to our previous works ${ }^{6,7,18,19}$. Here we specifically focused on highintensity stimuli $(5 \mathrm{~mA})$ delivered to the forepaw, due to their ability to maximize cortical activation, consistently reaching the hindpaw cortex ${ }^{10}$

Another group of animals $(n=10)$ was used for the pharmacological experiments, in which few drops of lidocaine ( $2 \%$ in saline) - a blocker of voltage-dependent sodium channels - were applied to both the sciatic and saphenous nerves near the dorsal roots ganglia on the left side of the body. With this approach it was possible to reproduce the same effects of the section in terms of total deafferentation of the hindpaw, avoiding traumatic damage. The block of axonal conduction was verified by the absence of any cortical response to very-high-intensity $(10 \mathrm{~mA})$ hindpaw stimuli. As soon as the effect of the blocked axonal conduction was observed, we performed the same stimulation protocol described previously. The effects of lidocaine typically started decaying within 2 hour after the blockade. The experimental protocol was applied several times until a complete recovery of cortical responses evoked by hindpaw stimuli was observed.

A third group of animal was used to perform sham experiments $(n=8)$, in which the animals were kept with both nerves intact for the entire duration of the experiment. Besides the absence of peripheral nerve injury and block of the axonal conduction, the experimental protocol was the same as in injured animals.

Data analysis. Local field potential (LFP) responses were obtained by averaging across stimuli the raw signals recorded from the electrodes. LFP response amplitude was evaluated as the absolute value of the negative peak in the average response. The rationale for using the LFP response as our main measure is three-fold: (i) LFPs maximize our ability to detect cortical responses at a population level, integrating membrane potentials, synaptic inputs and action potentials; (ii) in our experience the LFP response detected in the infragranular cortical layers is more robust than measures that are sensitive only to action potentials, likely because LFPs integrate over larger neural populations (and are not distorted by possible population spikes, which instead can introduce variability after band-pass filtering at high frequencies); (iii) the infragranular LFP response was the main measure used in our previous studies $^{6-8,18,19}$, which allows direct comparison between studies to be performed. The latter reason is also valid for our choice of measuring the LFP response peak, which is particularly sensitive to the local synchronous response of the cortical population around the electrode. We also mesured the responses of the rectified multi-unit activity (rMUA), obtained by band-pass filtering the raw LFP signals at high frequencies (300-3000 Hz), rectifying the resulting signal, and averaging across stimuli. The magnitude of rMUA responses was evaluated as the sum of the rMUA response values in the first $50 \mathrm{~ms}$ post-stimulus, after subtracting the background. Spontaneous activity was quantified with the mean rMUA during 150-s periods immediately before the stimulation protocols, similarly to our previous studies ${ }^{6-8,18,19}$.

Statistical analysis. The amplitudes of LFP responses were entered in a two-way mixed ANOVA, with GROUP as first factor with 3 levels between-subjects (injury, block and sham experiments) and TIME as second factor with 4 levels within-subjects (P0, P1, P2 and P3). We then performed three separate follow-up one-way ANOVAs for each experimental group, or pooling injury and block animals together, testing the TIME factor.

The mean rMUA during periods of spontaneous activity was entered into a threeway mixed ANOVA, with GROUP as first factor with 3 levels between-subjects (injury, block and sham experiments), TIME as second factor with 4 levels withinsubjects (P0, P1, P2 y P3) and CORTEX as third factor with 2 levels within-subjects (hindpaw and forepaw). We repeated the analyses on spontaneous activity with the following progressively less conservative approaches: we performed the same threeway ANOVA using only "injury" and "block" experiments (i.e. without "sham" experiments) to increase the power of the "time" factor; we performed two separate two-way ANOVAs on "injury" and "block" experiments; we performed four one-way ANOVAs on "injury" and "block" experiments, separately for hindpaw cortex and forepaw cortex in each experimental group separately. The mean rMUA before and after spinal cord injury was entered into a two-way repeated-measures ANOVA, with PRE-POST spinal lesion as first factor and CORTEX as second factor.

Dunnett test was used for all post-hoc comparisons. Additional comparisons between means were performed with paired t-test. Results were considered significant at $\mathrm{p}<0.05$.

1. Moore, C. I. et al. Referred phantom sensations and cortical reorganization after spinal cord injury in humans. Proc Natl Acad Sci U S A 97, 14703-8 (2000).

2. Simões, E. L. et al. Functional expansion of sensorimotor representation and structural reorganization of callosal connections in lower limb amputees. J Neurosci 32, 3211-20 (2012).

3. Klit, H., Finnerup, N. B. \& Jensen, T. S. Central post-stroke pain: clinical characteristics, pathophysiology, and management. Lancet Neurol 8, 857-68 (2009).

4. Wrigley, P. J. et al. Neuropathic pain and primary somatosensory cortex reorganization following spinal cord injury. Pain 141, 52-9 (2009).

5. Gustin, S. M. et al. Pain and plasticity: is chronic pain always associated with somatosensory cortex activity and reorganization? J Neurosci 32, 14874-84 (2012).

6. Aguilar, J. et al. Spinal cord injury immediately changes the state of the brain. J Neurosci 30, 7528-37 (2010). 
7. Humanes-Valera, D., Aguilar, J. \& Foffani, G. Reorganization of the intact somatosensory cortex immediately after spinal cord injury. PloS One 8, e69655 (2013).

8. Yagüe, J. G., Humanes-Valera, D., Aguilar, J. \& Foffani, G. Functional reorganization of the forepaw cortical representation immediately after thoracic spinal cord hemisection in rats. Exp Neurol 257, 19-24 (2014).

9. Moxon, K. A., Hale, L. L., Aguilar, J. \& Foffani, G. Responses of infragranular neurons in the rat primary somatosensory cortex to forepaw and hindpaw tactile stimuli. Neuroscience 156, 1083-92 (2008).

10. Morales-Botello, M. L., Aguilar, J. \& Foffani, G. Imaging the spatio-temporal dynamics of supragranular activity in the rat somatosensory cortex in response to stimulation of the paws. PloS One 7, e40174 (2012).

11. Merzenich, M. M. et al. Progression of change following median nerve section in the cortical representation of the hand in areas $3 \mathrm{~b}$ and 1 in adult owl and squirrel monkeys. Neuroscience 10, 639-65 (1983).

12. Wall, J. T. \& Cusick, C. G. Cutaneous responsiveness in primary somatosensory (S-I) hindpaw cortex before and after partial hindpaw deafferentation in adult rats. J Neurosci 4, 1499-515 (1984).

13. Calford, M. B. \& Tweedale, R. Immediate and chronic changes in responses of somatosensory cortex in adult flying-fox after digit amputation. Nature 332, 446-8 (1988).

14. Cusick, C. G., Wall, J. T., Whiting, J. H. \& Wiley, R. G. Temporal progression of cortical reorganization following nerve injury. Brain Res 537, 355-8 (1990).

15. Byrne, J. A. \& Calford, M. B. Short-term expansion of receptive fields in rat primary somatosensory cortex after hindpaw digit denervation. Brain Res $\mathbf{5 6 5}$, 218-24 (1991)

16. Faggin, B. M., Nguyen, K. T. \& Nicolelis, M. A. Immediate and simultaneous sensory reorganization at cortical and subcortical levels of the somatosensory system. Proc Natl Acad Sci U S A 94, 9428-33 (1997).

17. Paxinos, G. \& Watson, C. The rat brain in stereotaxic coordinates. (Academic Press, Amsterdam, 2007).

18. Aguilar, J. et al. Spinal direct current stimulation modulates the activity of gracile nucleus and primary somatosensory cortex in anaesthetized rats. J Physiol 589, 4981-96 (2011).

19. Yague, J. G., Foffani, G. \& Aguilar, J. Cortical hyperexcitability in response to preserved spinothalamic inputs immediately after spinal cord hemisection. Exp Neurol 227, 252-63 (2011)

20. Turnbull, B. G. \& Rasmusson, D. D. Chronic effects of total or partial digit denervation on raccoon somatosensory cortex. Somatosens Mot Res 8, 201-13 (1991).

21. Rasmusson, D. D., Webster, H. H. \& Dykes, R. W. Neuronal response properties within subregions of raccoon somatosensory cortex 1 week after digit amputation. Somatosens Mot Res 9, 279-89 (1992).

22. Jones, E. G. Cortical and subcortical contributions to activity-dependent plasticity in primate somatosensory cortex. Annu Rev Neurosci 23, 1-37 (2000).

23. Chen, R., Cohen, L. G. \& Hallett, M. Nervous system reorganization following injury. Neuroscience 111, 761-73 (2002).

24. Darian-Smith, C. \& Brown, S. Functional changes at periphery and cortex following dorsal root lesions in adult monkeys. Nat Neurosci 3, 476-81 (2000).

25. Ghosh, A. et al. Functional and anatomical reorganization of the sensory-motor cortex after incomplete spinal cord injury in adult rats. J Neurosci $29,12210-9$ (2009).

26. Ghosh, A. et al. Heterogeneous spine loss in layer 5 cortical neurons after spinal cord injury. Cereb Cortex 22, 1309-1317 (2012).
27. Yamahachi, H., Marik, S. A., McManus, J. N. J., Denk, W. \& Gilbert, C. D. Rapid axonal sprouting and pruning accompany functional reorganization in primary visual cortex. Neuron 64, 719-29 (2009).

28. Marik, S. A., Yamahachi, H., McManus, J. N. J., Szabo, G. \& Gilbert, C. D. Axonal dynamics of excitatory and inhibitory neurons in somatosensory cortex. PLoS Biol 8, e1000395 (2010)

29. Keck, T. et al. Loss of sensory input causes rapid structural changes of inhibitory neurons in adult mouse visual cortex. Neuron 71, 869-82 (2011)

30. Pluto, C. P., Chiaia, N. L., Rhoades, R. W. \& Lane, R. D. Reducing contralateral SI activity reveals hindlimb receptive fields in the SI forelimb-stump representation of neonatally amputated rats. J Neurophysiol 94, 1727-32 (2005).

31. Li, N. et al. Optogenetic-guided cortical plasticity after nerve injury. Proc Natl Acad Sci U S A 108, 8838-43 (2011).

32. Rasmusson, D. D., Louw, D. F. \& Northgrave, S. A. The immediate effects of peripheral denervation on inhibitory mechanisms in the somatosensory thalamus. Somatosens Mot Res 10, 69-80 (1993).

33. Pettit, M. J. \& Schwark, H. D. Receptive field reorganization in dorsal column nuclei during temporary denervation. Science 262, 2054-6 (1993).

34. Nicolelis, M. A., Lin, R. C., Woodward, D. J. \& Chapin, J. K. Induction of immediate spatiotemporal changes in thalamic networks by peripheral block of ascending cutaneous information. Nature 361, 533-6 (1993).

35. Fox, K., Wallace, H. \& Glazewski, S. Is there a thalamic component to experience dependent cortical plasticity? Philos Trans R Soc Lond B Biol Sci 357, 1709-15 (2002).

36. Aguilar, J. R. \& Castro-Alamancos, M. A. Spatiotemporal gating of sensory inputs in thalamus during quiescent and activated states. J Neurosci 25, 10990-1002 (2005).

\section{Acknowledgments}

This work was supported by Fundación para la Investigación Sanitaria en Castilla-La Mancha PI-2010/027 and funded by Ministerio de Economía y Competitividad, co-funded by FEDER SAF2012-40109. We thank Elena Alonso-Calviño and Elena Fernández-López for their technical help

\section{Author contributions}

The research was designed by D.H.-V., G.F. and J.A.; experiments were performed by D.H.-V.; data were analyzed by D.H.-V. and G.F.; wrote the manuscript D.H.-V., G.F., J.A.

\section{Additional information}

Competing financial interests: The authors declare no competing financial interests.

How to cite this article: Humanes-Valera, D., Foffani, G. \& Aguilar, J. Increased cortical responses to forepaw stimuli immediately after peripheral deafferentation of hindpaw inputs. Sci. Rep. 4, 7278; DOI:10.1038/srep07278 (2014)

This work is licensed under a Creative Commons Attribution-NonCommercialNoDerivs 4.0 International License. The images or other third party material in this article are included in the article's Creative Commons license, unless indicated otherwise in the credit line; if the material is not included under the Creative Commons license, users will need to obtain permission from the license holder in order to reproduce the material. To view a copy of this license, visit http:// creativecommons.org/licenses/by-nc-nd/4.0/ 